\title{
KIC 10080943: a binary star with two $\gamma$ Doradus $/ \delta$ Scuti hybrid pulsators. Analysis of the g modes
}

\author{
M. A. Keen ${ }^{1,2 \star}$, T. R. Bedding $g^{1,2} \dagger$, S. J. Murphy ${ }^{1,2} \ddagger$, V. S. $\operatorname{Schmid}^{3}$, C. Aerts ${ }^{3,4}$, \\ A. Tkachenko ${ }^{3}$, R.-M. Ouazzani ${ }^{2}$ and D.W. Kurtz ${ }^{5}$ \\ ${ }^{1}$ Sydney Institute for Astronomy, School of Physics, The University of Sydney, NSW 2006, Australia \\ ${ }^{2}$ Stellar Astrophysics Centre, Department of Physics and Astronomy, Aarhus University, Denmark \\ ${ }^{3}$ Institute of Astronomy, KU Leuven, Celestijnenlaan 200D, B - 3001 Leuven, Belgium \\ ${ }^{4}$ Department of Astrophysics, Radboud University Nijmegen, P.O. Box 9010, 6500 GL Nijmegen, The Netherlands \\ ${ }^{5}$ Jeremiah Horrocks Institute, University of Central Lancashire, Preston PR1 2HE, UK
}

14 September 2015

\begin{abstract}
We use four years of Kepler photometry to study the non-eclipsing spectroscopic binary KIC 10080943 . We find both components to be $\gamma$ Doradus/ $\delta$ Scuti hybrids, which pulsate in both $\mathrm{p}$ and $\mathrm{g}$ modes. We present an analysis of the g modes, which is complicated by the fact that the two sets of $\ell=1$ modes partially overlap in the frequency spectrum. Nevertheless, it is possible to disentangle them by identifying rotationally split doublets from one component and triplets from the other. The identification is helped by the presence of additive combination frequencies in the spectrum that involve the doublets but not the triplets. The rotational splittings of the multiplets imply core rotation periods of about $11 \mathrm{~d}$ and $7 \mathrm{~d}$ in the two stars. One of the stars also shows evidence of $\ell=2$ modes.
\end{abstract}

Key words: asteroseismology - binaries: spectroscopic - stars: individual: KIC 10080943 - stars: oscillations - stars: variables: $\delta$ Scuti - stars: variables: general

\section{INTRODUCTION}

Pulsating stars in binary systems have great potential to contribute to our understanding of stellar astrophysics. The orbit provides mass constraints for the two stars, while modelling of the pulsations (asteroseismology) yields information about their internal structure and rotation. When both stars are pulsating, such binary systems are particularly valuable.

Stars can oscillate in both pressure modes ( $p$ modes), which are most sensitive to conditions in the outer layers of the star, and gravity modes (g modes), which are most sensitive to the interior (e.g., Aerts, Christensen-Dalsgaard \& Kurtz 2010). Observations with the Kepler spacecraft have revealed that many main-sequence $\mathrm{A}$ and $\mathrm{F}$ stars show both $\delta$ Scuti (p-mode) and $\gamma$ Doradus (g-mode) pulsations (Grigahcène et al. 2010; Uytterhoeven et al. 2011; Tkachenko et al. 2013; Balona 2014).

Detailed studies of $\delta$ Sct $/ \gamma$ Dor hybrids have so far been carried out for two stars, namely KIC 11145123 (Kurtz et al.

\footnotetext{
* E-mail: mkee4218@uni.sydney.edu.au (MAK)

$\dagger$ E-mail: bedding@physics.usyd.edu.au (TRB)

$\ddagger$ E-mail: murphy@physics.usyd.edu.au (SJM)
}

2014) and KIC 9244992 (Saio et al. 2015). Both of those stars rotate slowly (periods of $100 \mathrm{~d}$ and $66 \mathrm{~d}$, respectively), with the rotation of the core differing only slightly from the rotation at the surface. The results showed that a strong angular momentum transport mechanism must be at work in main-sequence, intermediate-mass stars.

Here we discuss KIC 10080943, which is a non-eclipsing, double-lined spectroscopic binary (Tkachenko et al. 2013) in which we find both components to be $\gamma$ Dor $/ \delta$ Sct hybrids with moderate rotation. Huber et al. (2014) listed the following properties for KIC 10080943: $T_{\text {eff }}=7360 \pm 260 \mathrm{~K}$, $\log g=4.0 \pm 0.4$ and $[\mathrm{Fe} / \mathrm{H}]=-0.2 \pm 0.3$, while the visual magnitude was measured by Tkachenko et al. (2013) to be $V=11.7$.

This paper presents our analysis of the g-mode spectrum of KIC 10080943. A companion paper by Schmid et al. (2015) discusses the p modes and the binary properties, as well as addressing the individual stellar parameters of the constituent stars, as has been done for eclipsing binaries with intermediate-mass primaries (Hambleton et al. 2013; Debosscher et al. 2013; Maceroni et al. 2014). In particular, we have measured phase modulations in this system (Murphy et al. 2014; Murphy \& Shibahashi 2015), showing 
that orbital variations in the light arrival times of some of the p-mode pulsations occur in anti-phase with others. This demonstrates that both stars pulsate in $\mathrm{p}$ modes as discussed by Schmid et al. (2015). The same methodology cannot be applied to the g modes because the higher frequency density demands an unachievable frequency resolution, given the sampling required for the short orbit. Nonetheless, the focus of this paper is to show that both components also pulsate in g modes.

\section{DATA ANALYSIS \& RESULTS}

When analysing high-order g modes, we are guided by the expectation that they are equally spaced in period (Tassoul $1980)$. However, the steep composition gradient ( $\mu$ gradient) between the core and outer layers can cause deviations from this uniform period spacing (Miglio et al. 2008). Furthermore, as shown by Bouabid et al. (2013), stellar rotation causes the period spacing to vary with frequency. These effects have been observed in Kepler data for a number of $\gamma$ Dor stars (Bedding et al. 2014; Van Reeth et al. 2015a,b).

For KIC 10080943, we used long-cadence data (29.4minute sampling) from the full Kepler mission, spanning $1470.5 \mathrm{~d}(4.0 \mathrm{y})$. The Fourier spectrum (Fig. 1) shows pulsations in both the g-mode and p-mode frequency regions. These regions are clearly separated, with the g-mode region below approximately $6 \mathrm{~d}^{-1}$ and the p-mode region above approximately $8 \mathrm{~d}^{-1}$.

At low frequency there is a peak at $0.065209 \mathrm{~d}^{-1}$ with a long series of harmonics. Such harmonic series are often seen in binary stars. The inverse of this frequency is $15.335 \mathrm{~d}$, which we take as the binary orbital period. KIC 10080943 was already established as a binary by Tkachenko et al. (2013), and was selected for spectroscopic observations to determine the orbital parameters of the system. Those are presented in Schmid et al. (2015).

To analyse the pulsation content, we sequentially extracted the 250 strongest peaks in the frequency range from zero to the Nyquist frequency $\left(24.47 \mathrm{~d}^{-1}\right)$ using PERIOD04 (Lenz \& Breger 2004, 2005). Non-linear least-squares fitting was implemented during extraction. Although we were interested in the g modes, the p modes were also extracted because the spectral windows of the $\mathrm{p}$ and $\mathrm{g}$ modes overlap. We also checked that the extracted peaks were not Nyquist aliases using the method outlined by Murphy, Shibahashi \& Kurtz (2013).

In the range 0.6 to $1.7 \mathrm{~d}^{-1}$ we identified six series of pulsation modes. Each is approximately equally spaced in period, consistent with a series of overtones with a common $\ell$ and $m$ value. Furthermore, some of the series are separated from others by a fixed frequency, suggesting rotational splitting. Given that KIC 10080943 is a binary system, we were led to identify two sets of g modes with slightly different period spacings, one from each component of the binary. This is shown in Figs 2 and 3, where we have arbitrarily labelled the components of the binary as Stars A and B. In Star A we see rotationally split doublets, while Star B has triplets in which the central component is weak. There is also a sequence with slightly lower period spacing (asterisks) that we tentatively identify with $\ell=2$ modes. Note that Bed-

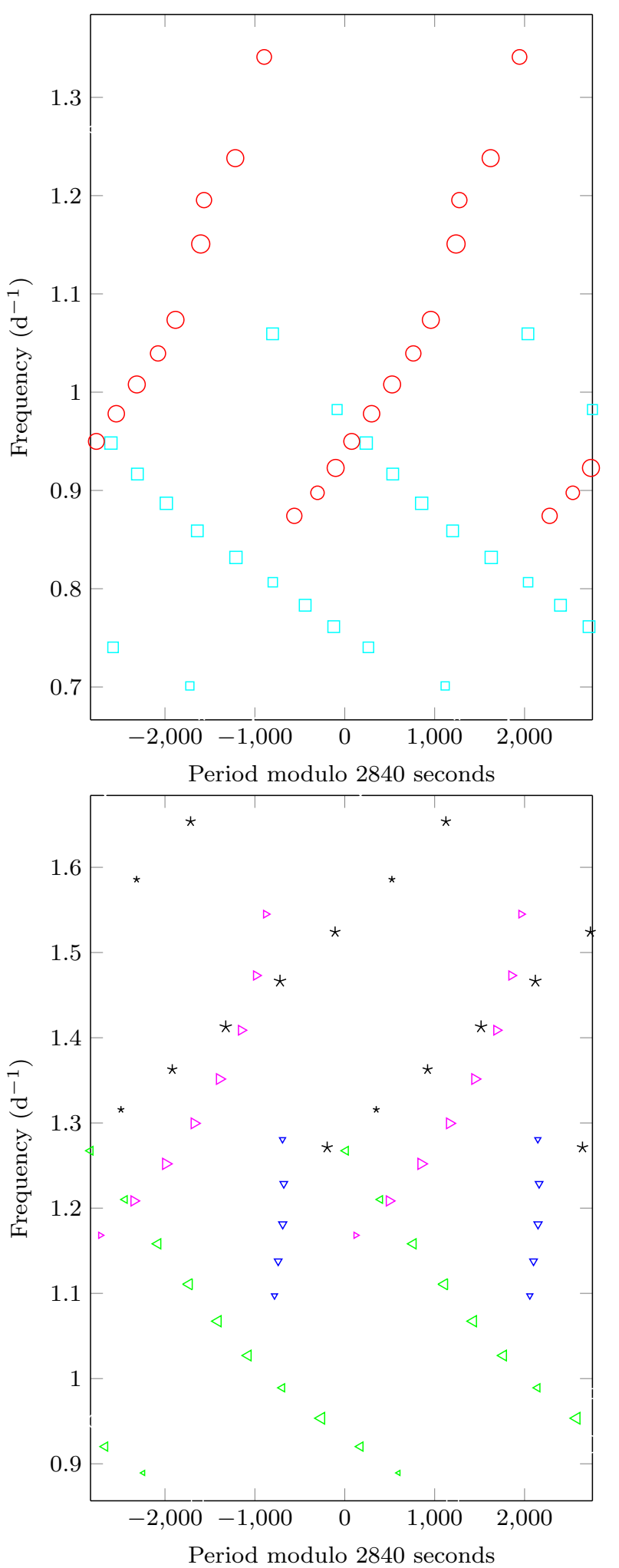

Figure 4. Period échelle diagrams of the g modes in KIC 10080943, for Star A (upper) and Star B (lower). The data have been plotted twice for clarity. The symbol size is proportional to the logarithm of the amplitude of the corresponding peak in the Fourier spectrum. 


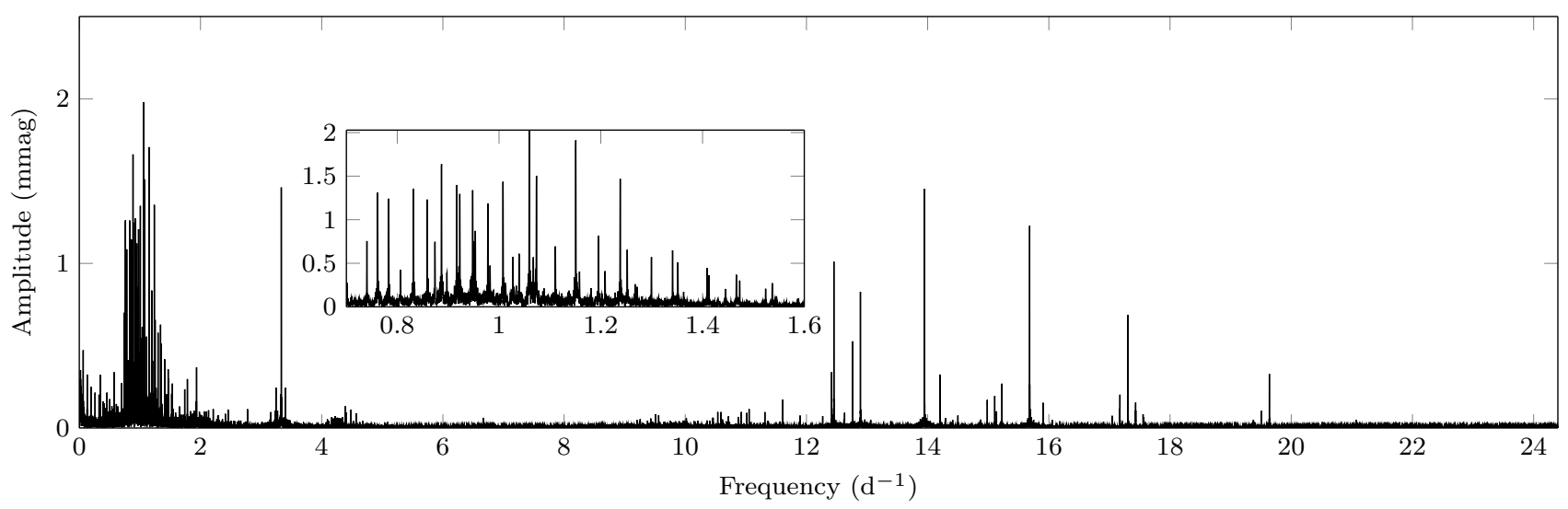

Figure 1. Fourier amplitude spectrum of KIC 10080943 from Kepler long cadence data, showing the clearly separated g-mode and p-mode regions. Inset: close-up of the g modes.
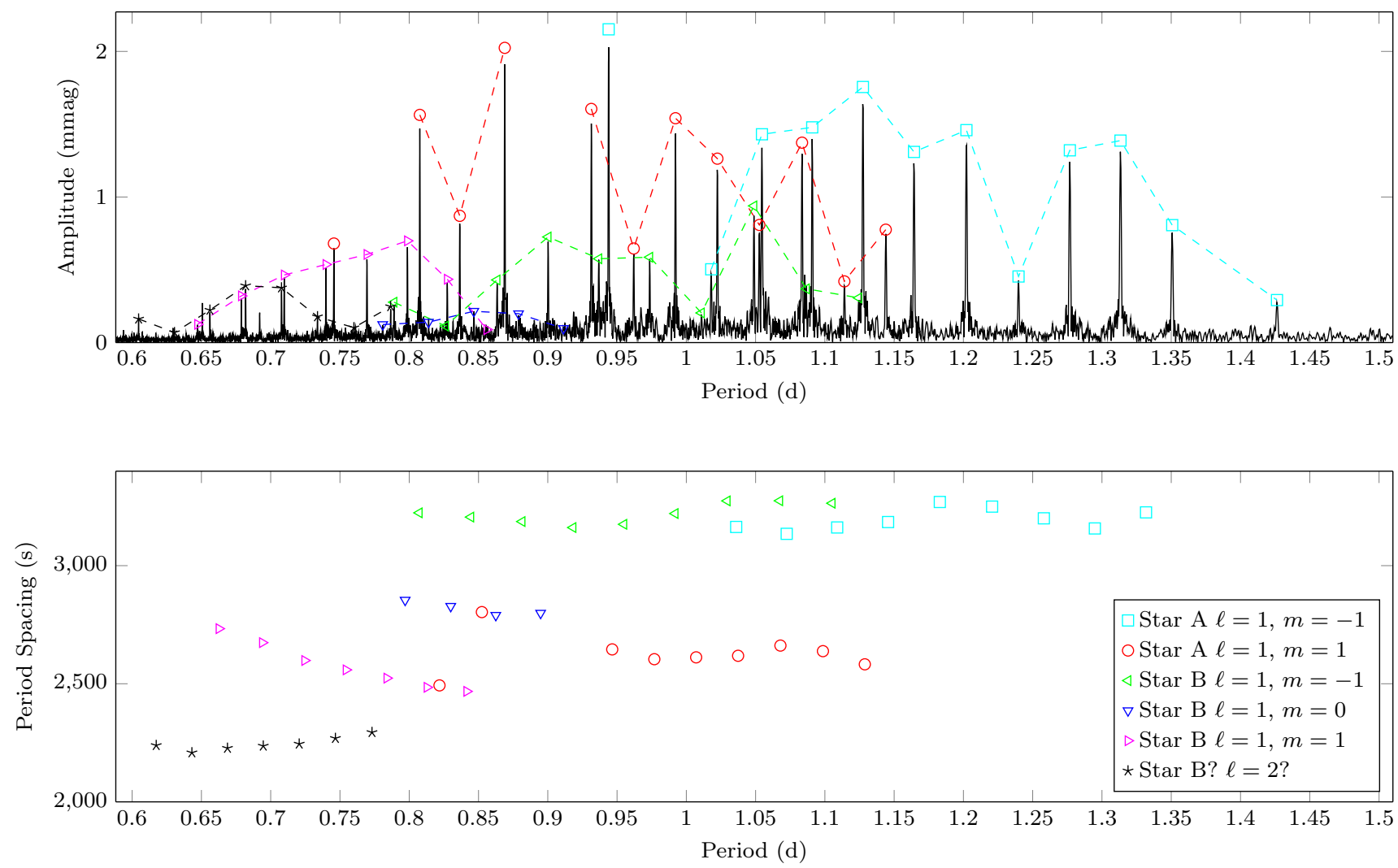

Figure 2. Top: Amplitude spectrum of the g-mode region, plotted as a function of period, showing the identified peaks. Dashed lines connect neighbouring peaks in the same g-mode series. Bottom: Period spacings between adjacent modes in each series.

ding et al. (2014) have identified clear evidence for $\ell=2$ g modes in another $\gamma$ Doradus star, KIC 3127996.

The peaks are plotted in échelle format in Fig. 4 for the two stars, with symbol size indicating amplitude. The frequencies and amplitudes for the six g-mode series are given in Tables 1 to 3 . Importantly, all significant peaks in this frequency range have been identified with modes. The number of points in the échelle diagram (Fig. 4) is greater than the number in the period spacing diagram (Fig. 2), since the latter require two neighbouring modes for each period spac- ing, but some modes are missing. Values of $m$ could still be assigned to modes in incomplete multiplets by tracing their period spacings.

\subsection{Combination Frequencies}

Kurtz et al. (2015) showed recently that the Fourier spectra of some g-mode pulsators are dominated by combination frequencies. We have checked whether any peaks in the main g-mode region of KIC 10080943 (0.6 to $\left.1.7 \mathrm{~d}^{-1}\right)$ are 


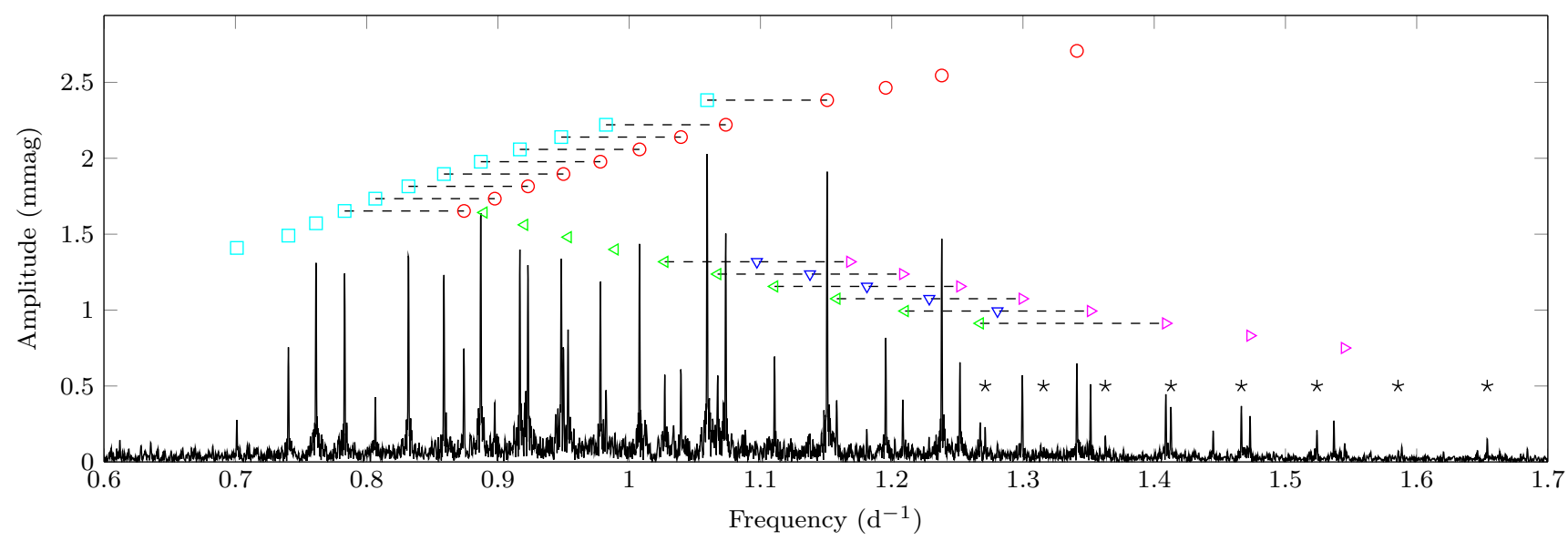

Figure 3. Identification of rotationally split g-mode multiplets in KIC 10080943. Dashed lines connect peaks in each multiplet. Colours and symbols used match those of Fig. 2.

combinations of other peaks. This was done by creating linear combinations (including harmonics) of the 15 strongest peaks, using up to three of these peaks with integer coefficients from -3 to 3 . None of the observed frequencies were found to be within $1 / T=6.8 \times 10^{-4} \mathrm{~d}^{-1}$ of a calculated combination frequency. This is not surprising, given that all these peaks have already been identified with one of the six series of overtones.

On the other hand, the small hump in the frequency range 4.0 to $4.8 \mathrm{~d}^{-1}$ is made up of combination peaks. To be specific, these peaks have frequencies $f_{3.3}+f_{g}$, where $f_{3.3}=3.3334962 \pm 0.0000017 \mathrm{~d}^{-1}$ is one of the strongest peaks in the amplitude spectrum. As can be seen in Fig. 5, the doublets we have assigned to Star A couple with $f_{3.3}$ to produce additive combination frequencies, but the triplets we have assigned to Star B do not. This suggests that $f_{3.3}$ originates in Star A and is further evidence that g-mode pulsations occur in both components of the binary. Subtractive $\left(f_{3.3}-f_{g}\right)$ combinations are also present.

\section{$2.2 \quad$ Rotational Splittings}

As discussed above, we identified two series of rotationally split $\ell=1$ multiplets, one in each component of the binary see Fig. 3. The triplets in Star B $(m=0, \pm 1)$ have frequency splittings of about $0.07 \mathrm{~d}^{-1}$ and are slightly asymmetric, as shown in the lower panel of Fig. 6. The asymmetry is small, at about 0.5 per cent, and is presumably caused by secondorder effects (see Murphy 2014 for a discussion of non-equal rotational splittings in A stars in the Kepler era). The implied rotation period at the edge of the convective core is about $7 \mathrm{~d}$.

The doublets in Star A, which presumably correspond to azimuthal orders $m= \pm 1$, have a frequency splitting of $0.091 \mathrm{~d}^{-1}$. This varies slightly with frequency, as shown in the upper panel of Fig. 6. This variation cannot be explained by radial differential rotation because the g-modes all probe the same region outside the core. Instead, it reflects the fact that the Ledoux constant $C_{n, l}$ has a small dependence on radial order, $n$ (Bedding et al., in prep.). Star A is a partic-
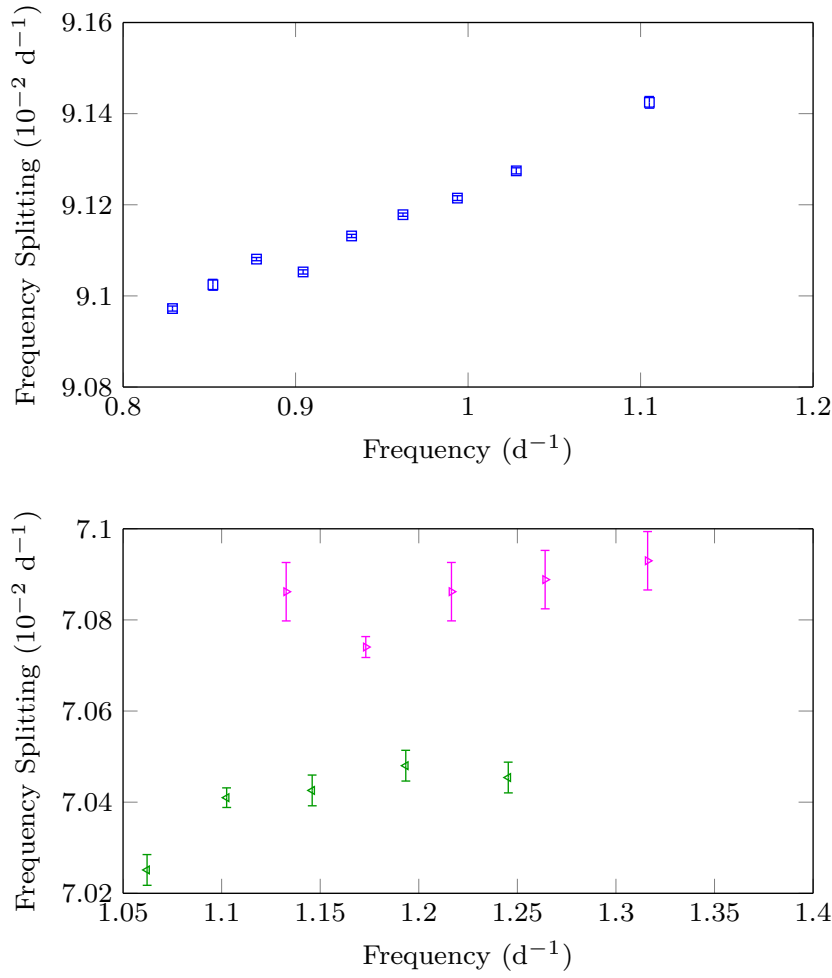

Figure 6. Upper panel: rotational splittings of the doublets in Star A, measured between the $m=-1$ and $m=1$ modes for each radial order. Lower panel: rotational splittings of the triplets for Star B. The upper sequence (magenta symbols) show the splittings measured between $m=0$ and $m=1$, while the lower sequence (green symbols) show the splittings between $m=-1$ and $m=0$.

ularly good example of this phenomenon. Its core rotation period is about $11 \mathrm{~d}$. 


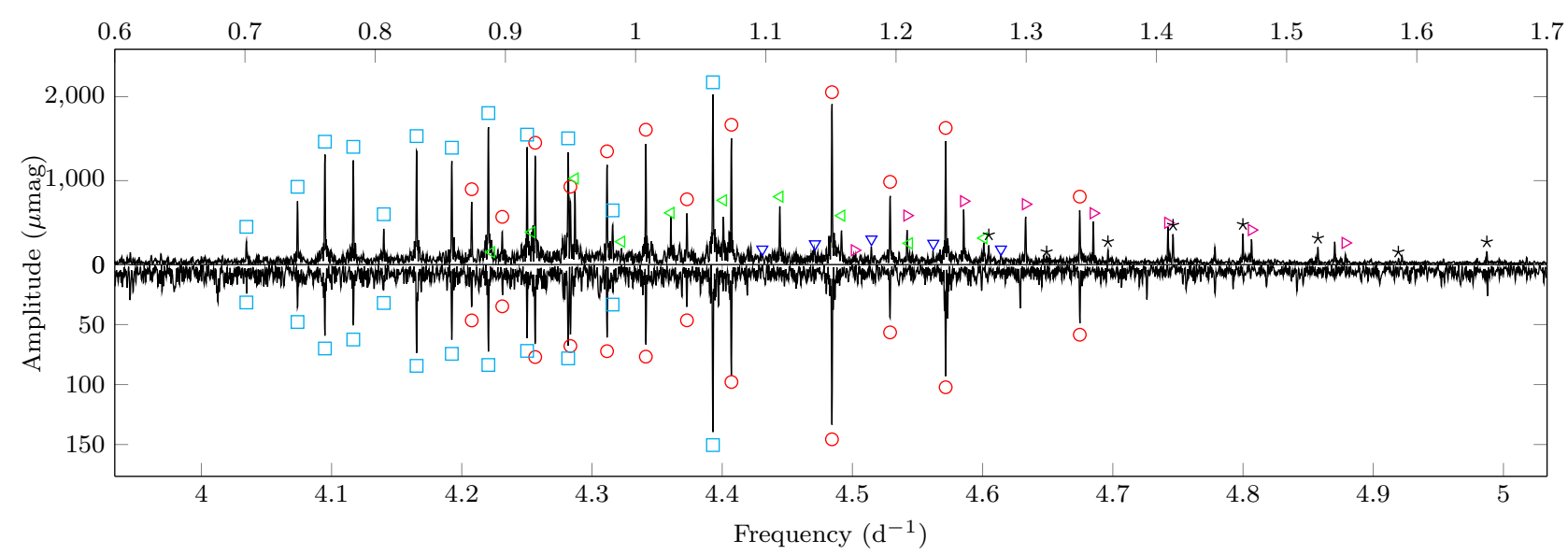

Figure 5. Additive combination frequencies in KIC 10080943. The upper plot shows the g-mode region of the amplitude spectrum while the lower plot shows a higher-frequency section of exactly the same length, with an offset of $f_{3.3}=3.33 \mathrm{~d}^{-1}$. Coloured symbols have the same meanings as in previous figures, with the addition that those in the lower panel indicate combination frequencies. Note the different amplitude scales in the two panels.

\section{CONCLUSION}

KIC 10080943 is a binary system with two pulsating components, making it particularly interesting for asteroseismology. The combined oscillation spectrum is complicated because the two sets of $\mathrm{g}$ modes partially overlap in frequency. However, it was possible to disentangle them by identifying rotationally split doublets in Star A and triplets in Star B. The identification was helped by the presence of additive combination frequencies in Star A and not Star B. The rotational splittings of the g-mode multiplets imply core rotation periods of about $11 \mathrm{~d}$ and $7 \mathrm{~d}$ in Stars A and B, respectively. Analysis of the p modes, together with models of the oscillation frequencies, are presented by Schmid et al. (2015) and in future papers.

\section{ACKNOWLEDGEMENTS}

We gratefully acknowledge the entire Kepler team for providing such superb data. This research was supported by the Australian Research Council. Funding for the Stellar Astrophysics Centre is provided by the Danish National Research Foundation (grant agreement no.:DNRF106). The research is supported by the ASTERISK project (ASTERoseismic Investigations with SONG and Kepler) funded by the European Research Council (grant agreement no.: 267864). VAS is an Aspirant PhD Fellow and AT is a Postdoctoral Fellow of the Fund for Scientific Research of Flanders (FWO), Belgium.

\section{REFERENCES}

Aerts C., Christensen-Dalsgaard J., Kurtz D. W., 2010, Asteroseismology, Astronomy and Astrophysics Library. Springer

Balona L. A., 2014, MNRAS, 437, 1476

Bedding T. R., Murphy S. J., Colman I. L., Kurtz D. W., 2014, arXiv:1411.1883
Table 1. A least-squares fit of the g-mode $\ell=1$ doublets in Star A (as shown in Fig. 3) for KIC 10080943. The uncertainties were determined by non-linear least-squares fitting.

\begin{tabular}{|c|c|c|}
\hline $\mathrm{m}$ & $\begin{array}{l}\text { Frequency } \\
\quad\left(d^{-1}\right)\end{array}$ & $\begin{array}{c}\text { Amplitude } \\
(\mathrm{mmag}) \pm 0.007\end{array}$ \\
\hline-1 & $0.701089 \pm 0.000011$ & 0.237 \\
\hline-1 & $0.740343 \pm 0.000003$ & 0.726 \\
\hline-1 & $0.761387 \pm 0.000002$ & 1.314 \\
\hline-1 & $0.783179 \pm 0.000002$ & 1.163 \\
\hline 1 & $0.874151 \pm 0.000003$ & 0.762 \\
\hline-1 & $0.806578 \pm 0.000006$ & 0.440 \\
\hline 1 & $0.897602 \pm 0.000006$ & 0.415 \\
\hline-1 & $0.831815 \pm 0.000002$ & 1.414 \\
\hline 1 & $0.922896 \pm 0.000002$ & 1.436 \\
\hline-1 & $0.858849 \pm 0.000002$ & 1.263 \\
\hline 1 & $0.949902 \pm 0.000003$ & 0.971 \\
\hline-1 & $0.886926 \pm 0.000002$ & 1.679 \\
\hline 1 & $0.978058 \pm 0.000002$ & 1.239 \\
\hline-1 & $0.916677 \pm 0.000002$ & 1.401 \\
\hline 1 & $1.007856 \pm 0.000002$ & 1.379 \\
\hline-1 & $0.948214 \pm 0.000002$ & 1.474 \\
\hline 1 & $1.039429 \pm 0.000003$ & 0.731 \\
\hline-1 & $0.982322 \pm 0.000005$ & 0.511 \\
\hline 1 & $1.073596 \pm 0.000002$ & 1.493 \\
\hline-1 & $1.059381 \pm 0.000012$ & 2.169 \\
\hline 1 & $1.150806 \pm 0.000001$ & 1.956 \\
\hline-1 & $1.110715 \pm 0.000004$ & 0.709 \\
\hline 1 & $1.195443 \pm 0.000003$ & 0.815 \\
\hline 1 & $1.238138 \pm 0.000002$ & 1.455 \\
\hline 1 & $1.341122 \pm 0.000004$ & 0.671 \\
\hline
\end{tabular}

Bouabid M., Dupret M., Salmon S., Montalbán J., Miglio A., Noels A., 2013, MNRAS, 429, 2500

Debosscher J. et al., 2013, A\&A, 556, A56

Grigahcène A. et al., 2010, ApJ, 713, L192 
Table 2. A least-squares fit of the g-mode $\ell=1$ triplets in Star B (as shown in Fig. 3) for KIC 10080943. The uncertainties were determined by non-linear least-squares fitting.

\begin{tabular}{rcc}
\hline $\mathrm{m}$ & $\begin{array}{c}\text { Frequency } \\
\left(\mathrm{d}^{-1}\right)\end{array}$ & $\begin{array}{c}\text { Amplitude } \\
(\mathrm{mmag}) \pm 0.007\end{array}$ \\
\hline-1 & $0.889276 \pm 0.000051$ & 0.048 \\
\hline-1 & $0.920191 \pm 0.000009$ & 0.286 \\
\hline-1 & $0.953444 \pm 0.000003$ & 0.926 \\
\hline-1 & $0.989185 \pm 0.000015$ & 0.173 \\
\hline-1 & $1.027054 \pm 0.000005$ & 0.516 \\
0 & $1.097305 \pm 0.000029$ & 0.082 \\
1 & $1.168167 \pm 0.000035$ & 0.071 \\
\hline-1 & $1.067338 \pm 0.000004$ & 0.665 \\
0 & $1.137748 \pm 0.000018$ & 0.145 \\
1 & $1.208489 \pm 0.000005$ & 0.484 \\
\hline-1 & $1.110715 \pm 0.000004$ & 0.709 \\
0 & $1.181141 \pm 0.000013$ & 0.202 \\
1 & $1.252003 \pm 0.000004$ & 0.655 \\
\hline-1 & $1.158160 \pm 0.000005$ & 0.483 \\
0 & $1.228640 \pm 0.000016$ & 0.151 \\
1 & $1.299529 \pm 0.000004$ & 0.619 \\
\hline-1 & $1.210161 \pm 0.000017$ & 0.153 \\
0 & $1.280615 \pm 0.000030$ & 0.082 \\
1 & $1.351545 \pm 0.000005$ & 0.512 \\
\hline-1 & $1.267386 \pm 0.000012$ & 0.215 \\
1 & $1.408809 \pm 0.000006$ & 0.398 \\
\hline 1 & $1.473037 \pm 0.000008$ & 0.312 \\
\hline 1 & $1.545034 \pm 0.000016$ & 0.158 \\
\hline & &
\end{tabular}

Table 3. A least-squares fit of the series of g-modes of KIC 10080943 marked with black asterisks in Figs 2 to 4 . These peaks are tentatively labelled as having $\ell=2$ and belonging to Star B. The uncertainties were determined by non-linear leastsquares fitting.

\begin{tabular}{cc}
\hline $\begin{array}{c}\text { Frequency } \\
\left(\mathrm{d}^{-1}\right)\end{array}$ & $\begin{array}{c}\text { Amplitude } \\
(\mathrm{mmag}) \pm 0.007\end{array}$ \\
\hline $1.271255 \pm 0.000010$ & 0.254 \\
$1.315665 \pm 0.000050$ & 0.051 \\
$1.362751 \pm 0.000015$ & 0.173 \\
$1.412779 \pm 0.000007$ & 0.369 \\
$1.466406 \pm 0.000007$ & 0.378 \\
$1.524018 \pm 0.000012$ & 0.214 \\
$1.585784 \pm 0.000050$ & 0.050 \\
$1.653745 \pm 0.000015$ & 0.170 \\
\hline
\end{tabular}

Hambleton K. M. et al., 2013, MNRAS, 434, 925

Huber D. et al., 2014, ApJS, 211, 2

Kurtz D. W., Saio H., Takata M., Shibahashi H., Murphy S. J., Sekii T., 2014, MNRAS, 444, 102

Kurtz D. W., Shibahashi H., Murphy S. J., Bedding T. R., Bowman D. M., 2015, MNRAS, 450, 3015

Lenz P., Breger M., 2004, in IAU Symposium, Vol. 224, The A-Star Puzzle, Zverko J., Ziznovsky J., Adelman S. J., Weiss W. W., eds., pp. 786-790

Lenz P., Breger M., 2005, Communications in Asteroseismology, 146, 53

Maceroni C. et al., 2014, A\&A, 563, A59
Miglio A., Montalbán J., Noels A., Eggenberger P., 2008, MNRAS, 386, 1487

Murphy S. J., 2014, PhD thesis, Univ. of Central Lancashire

Murphy S. J., Bedding T. R., Shibahashi H., Kurtz D. W., Kjeldsen H., 2014, MNRAS, 441, 2515

Murphy S. J., Shibahashi H., 2015, MNRAS, 450, 4475

Murphy S. J., Shibahashi H., Kurtz D. W., 2013, MNRAS, 430, 2986

Saio H., Kurtz D. W., Takata M., Shibahashi H., Murphy S. J., Sekii T., Bedding T. R., 2015, MNRAS, 447, 3264 Schmid V. S. et al., 2015, ArXiv e-prints

Tassoul M., 1980, ApJS, 43, 469

Tkachenko A. et al., 2013, A\&A, 556, A52

Uytterhoeven K. et al., 2011, A\&A, 534, A125

Van Reeth T. et al., 2015a, A\&A, 574, A17

Van Reeth T. et al., 2015b, ApJS, 218, 27

This paper has been typeset from a $\mathrm{T}_{\mathrm{E}} \mathrm{X} / \mathrm{LAT}_{\mathrm{E}} \mathrm{X}$ file prepared by the author. 